\title{
Electron Density Geometry and the Quantum Theory of Atoms in Molecules
}

\author{
Timothy R. Wilson ${ }^{*}$ Anastassia N. Alexandrova ${ }^{\dagger}$ M.E. Eberhart ${ }^{* \neq}$
}

October 18, 2021

\begin{abstract}
A novel form of charge density analysis, that of isosurface curvature redistribution, is formulated and applied to the toy problem of carbonyl oxygen activation in formaldehyde. The isosurface representation of the electron charge density allows us to incorporate the rigorous geometric constraints of closed surfaces towards the analysis and chemical interpretation of the charge density response to perturbations. Visual inspection of 2D isosurface motion resulting from applied external electric fields reveals how isosurface curvature flows within and between atoms, and that a molecule can be uniquely and completely partitioned into chemically significant regions of positive and negative curvature. These concepts reveal that carbonyl oxygen activation proceeds primarily through curvature and charge redistribution within rather than between Bader atoms. Using gradient bundle analysis - the partitioning of formaldehyde into infinitesimal volume elements bounded by QTAIM zero flux surfaces - the observations from visual isosurface inspection are verified. The results of the formaldehyde carbonyl analysis are then shown to be transferable to the substrate carbonyl in the ketosteroid isomerase enzyme, laying the groundwork for extending this approach to the problems of enzymatic catalysis.

Keywords: QTAIM | GBA | electron density geometry | carbonyl activation

\footnotetext{
*Department of Chemistry, Colorado School of Mines, Golden CO, USA

†Department of Chemistry, UCLA

‡meberhar@mines.edu
} 


\section{Introduction}

The Quantum Theory of Atoms in Molecules (QTAIM) attributes special significance to volumes bounded by zero flux surfaces (ZFS), that is, surfaces through which the flux of the gradient of the electron charge density, $\rho(r)$, is everywhere zero [1-3]. These volumes possess well defined energies and hence unambiguous energy-related properties. While there are an infinite number of boundary conditions giving rise to such energetically unambiguous volumes [4-6], each imposes a different geometry on $\rho(r)$. In the case of the ZFS of QTAIM, the geometry imposed is that intrinsic to the electron density gradient, $\nabla \rho(r)$, or its dual representation as a set of nested isosurfaces. Keeping with the original vision for QTAIM as an exact chemical formalism rooted in the observable electron density [7], molecular properties should be seen as the consequential and quantifiable changes to this geometry due to chemical or physical perturbations.

Here, using an important problem drawn from carbonyl chemistry [8], we show that much of our empirically tuned "chemical intuition" regarding this process derives from mathematically rigorous geometrical constraints limiting the response of $\rho(r)$.

\section{Geometry of the electron density}

Electronic charge density is often represented with a set of simple closed isosurfaces that may, in turn, be pictured as contours in selected cut planes. Such representations have proved useful as a way to depict a molecule's response to chemical or physical perturbations. It is seldom noted, however, that in addition to chemical factors limiting this response there are geometric principles that further constrain the allowed changes to these surfaces.

The geometry of a surface in the neighborhood of a point is determined by its two principal curvatures at that point $\left(\kappa_{1}\right.$ and $\left.\kappa_{2}\right)$. The surface's total curvature is given either by its mean curvature $\left(\kappa_{1}+\kappa_{2}\right)$ or its Gaussian curvature $\left(\kappa_{1} \kappa_{2}\right)$. A point on a surface where both principal curvatures are positive is called an elliptic point, and near this point the surface is convex. If both principal curvatures are negative, the surface is said to be parabolic and is locally concave. Around a point where one principal curvature is negative and the other is positive the surface is saddle shaped and the point is called hyperbolic. Finally, about a point where at least one principal curvature vanishes, the surface is topologically flat.

Any closed $\rho(r)$ isosurface, may be decomposed into continuous regions in which every 

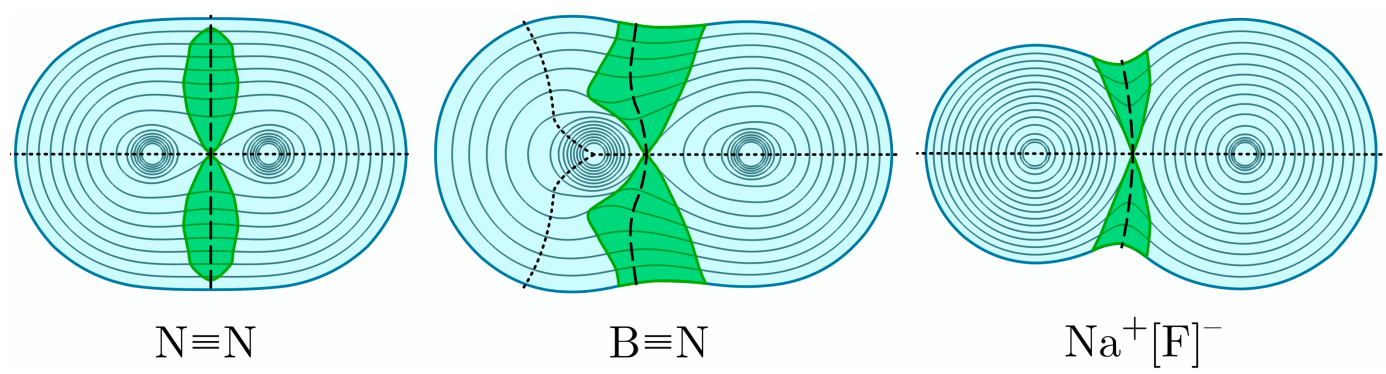

Figure 1. The corner (cyan) and trough (green) regions of the $\mathrm{N}_{2}, \mathrm{BN}$ and $\mathrm{NaF}$ molecules superimposed on charge density contour diagrams. Corner gradient paths are shown with light dashed black lines and the interatomic surface with heavy dashed black lines. Note that the boundary between the trough and corner regions is coincident with a line along which isosurface Gaussian curvature vanishes, i.e., at inflection points along the contours.

point is either elliptic (convex), parabolic (concave) or hyperbolic (saddle). The boundary between these regions occurs where one principal curvature vanishes and hence the Gaussian curvature is zero. In each such region there is at least one $2 \mathrm{D}$ critical point $(\mathrm{CP})$ where the Gaussian curvature achieves its extreme values. We designate these as convex, saddle, and concave CPs; the respective 2D counterparts of the $3 \mathrm{D}$ nuclear, bond/ring and cage CPs of QTAIM.

Generally, for nested isosurfaces where the field is smoothly varying, the convex, concave and saddle topological regions jointly create $3 \mathrm{D}$ regions. The isosurfaces of convex regions stack to form what are called corners. The locus of the convex CPs on corner isosurfaces lie along the gradient lines that intersect the isosurfaces where they are most curved; we call these corner lines, which are often coincident with QTAIM bond paths.

Stacked hyperbolic regions give rise to 3D structures we call troughs. The locus of CPs in these regions define trough lines. These lines lie along the boundaries between two bound atoms and terminate at $3 \mathrm{D}$ bond $\mathrm{CPs}$.

Stacked concave regions we call depressions, which are associated with the 3D-structure of the charge density intrinsic to rings of bound atoms. The locus of concave CPs gives rise to a depression line that terminate at ring CPs.

As examples, Figure 1 shows the charge density contour diagrams in planes containing the internuclear axis for the series of dimers $\mathrm{N}_{2}, \mathrm{BN}$ and $\mathrm{NaF}$, with trough and corner regions indicated. Due to their axial symmetry, isosurfaces may be generated by revolving individual contour lines about the internuclear axis. Note that in all cases the interatomic surface lies entirely within the trough region and bond paths are coincident with corner lines. However, the corner lines endow the density with additional structure in the non-bonding region. Even 
so, there is a boundary of zero Gaussian curvature (ZGC) separating the troughs from the corner regions. The shape of the troughs is distinctive and in some respects is indicative of the nature of the interaction between the bound atoms. For example, total negative curvature along the trough line increases with ionicity, or more precisely, with increasing spherical character of the bound atoms.

Changes to a molecule's external potential will necessarily alter isosurface curvature. However, the extent of these alterations must be consistent with the requirement that the total Gaussian curvature of any simple closed surface is $4 \pi$. Thus, for example, perturbations that increase the saddle character of a surface must be offset by regions of increased convexity. As it is common to picture the charge density in a plane as a series of contours, it is useful to generalize to closed curves. In this case, regardless of its shape, the integrated curvature around a closed contour line will be $2 \pi$. The total curvature along any segment of the curve can be determined by the angle of intersection of the tangent to the curve at the endpoints of the segment.

In addition to the geometric constraints acting on individual isosurfaces and contours, the form of the charge density imposes constraints on the character of nested isosurfaces. These effects are most obvious near a bond CP. To briefly explain: To second order the shape of the charge density around a bond CP can be written as $[1,9,10]$,

$$
\rho(r)-\rho\left(r_{b c p}\right)=\frac{1}{2}\left(\rho_{z z} z^{2}-\rho_{x x} x^{2}-\rho_{y y} y^{2}\right) \text {. }
$$
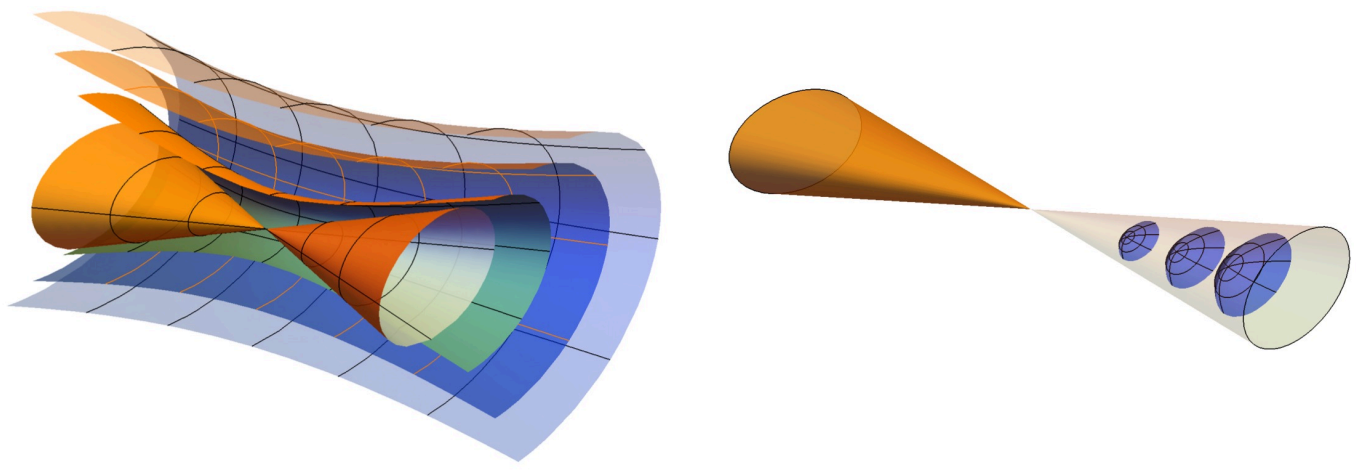

Figure 2. The isosurfaces near a bond CP. The isosurface passing through the bond point will have the form of an elliptic cone, with the bond path coincident with its axis. This cone is the asymptotic boundary separating the exterior isosurfaces (left) from those interior to the cone (right). The exterior isosurfaces, being hyperbolic, characterize a charge density trough. Typically, the trough line lies along the internuclear boundary. The interior isosurfaces are convex and define a charge density corner, with the corner line coincident with the bond path. 
Where the bond CP serves as the origin; $\rho\left(r_{b c p}\right)$ is the value of the charge density at the bond CP; $x, y$ and $z$ are the eigenvectors of the Hessian of $\rho(r)$; and $\rho_{x x}, \rho_{y y}$ and $\rho_{z z}$ are the magnitudes of the corresponding eigenvalues, e.g., $\rho_{x x}=\left|\frac{\partial^{2} \rho(r)}{\partial x^{2}}\right|$. We take $z$ to be the direction parallel to the internuclear axis and hence $\rho_{z z}$ is the positive eigenvalue while $x$ and $y$ are the directions of the negative eigenvalues.

When $\rho(r)-\rho\left(r_{b p}\right)$ is positive, Equation 1 represents nested hyperboloids of two sheets. When it is negative, it is the equation for nested hyperboloids of one sheet. And when $\rho(r)-$ $\rho\left(r_{b p}\right)=0$ (the isosurface passing through the bond CP), it is the equation for a double elliptic cone to which the two sets of hyperboloids are asymptotic (see Figure 2).

The cone passing through the bond $\mathrm{CP}$ is fully characterized by its two exterior angles $\theta$ and $\phi$ (Figure 3), with $\tan (\theta)=\sqrt{\frac{\rho_{y y}}{\rho_{z z}}}$ and $\tan (\phi)=\sqrt{\frac{\rho_{x x}}{\rho_{z z}}}$. The curvatures of the isosurfaces exterior to the cone are mediated by these angles, a perturbation that decreases $\frac{\rho_{y y}}{\rho_{z z}}$ and/or $\frac{\rho_{x x}}{\rho_{z z}}$ will necessarily increase the curvature of these isosurfaces. In a reciprocal way, there must be a corresponding increase to the interior angles of the cone that will necessitate a decrease in the curvature of the isosurface interior to the cone (see Figure 3). 

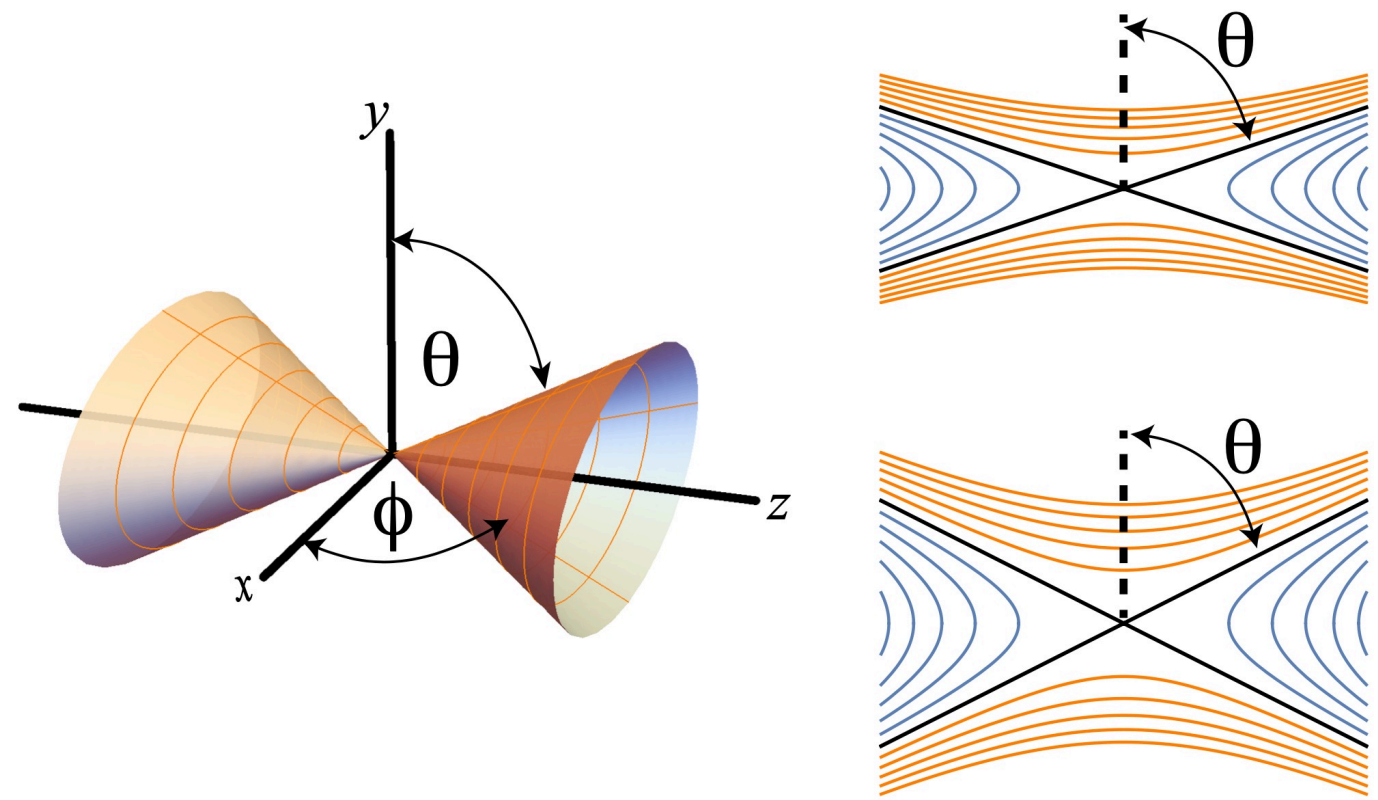

Figure 3. The isosurface sufficiently close to the bond CP will be well approximated by a double cone fully described by the angles $\theta$ and $\phi$. In any plane containing the bond path (the cone axis) the electron density contour lines will appear as represented on the right, where the orange contour lines are those of the hyperbolic trough region, the blue contour lines are those of the corner region, and the black contours are those of the double cone. As the angle $\theta$ decreases (going from the top set of contours to the bottom set), along the trough line the contours become more curved, while along the corner line the contours become less curved.

The key points here are: 1) The charge density of any molecule may be partitioned into space filling corner, trough, and depression regions separated by ZGC boundaries. 2) Troughs, corners and depressions are chemically significant structures that are characterized by isosurface curvature; 3) The total Gaussian curvature of a simple closed isosurface is $4 \pi$ and as an axiom the total curvature of a closed charge density contour is $2 \pi$; and 4) The curvature of corner regions e.g., along a bond path, are reciprocally tied to the curvature of trough regions perpendicular to the bond path.

\section{The Geometry of Carbonyl Activation}

As an example application, we consider the geometry of $\rho(r)$ of a carbonyl group and its change due to various external potentials. Carbonyl chemistry is a massive subject area [8] applicable to both natural [11] and human made systems [12-14]. Our particular interest is motivated in part by the ubiquity with which carbonyl activation is employed as a step in enzymatic pathways, e.g. carbonyl activation is done by peptidases and $\beta$-lactamases to facilitate the nucleophilic attack during the hydrolyses of the corresponding peptide and 

steroid molecule to facilitate its isomerization.

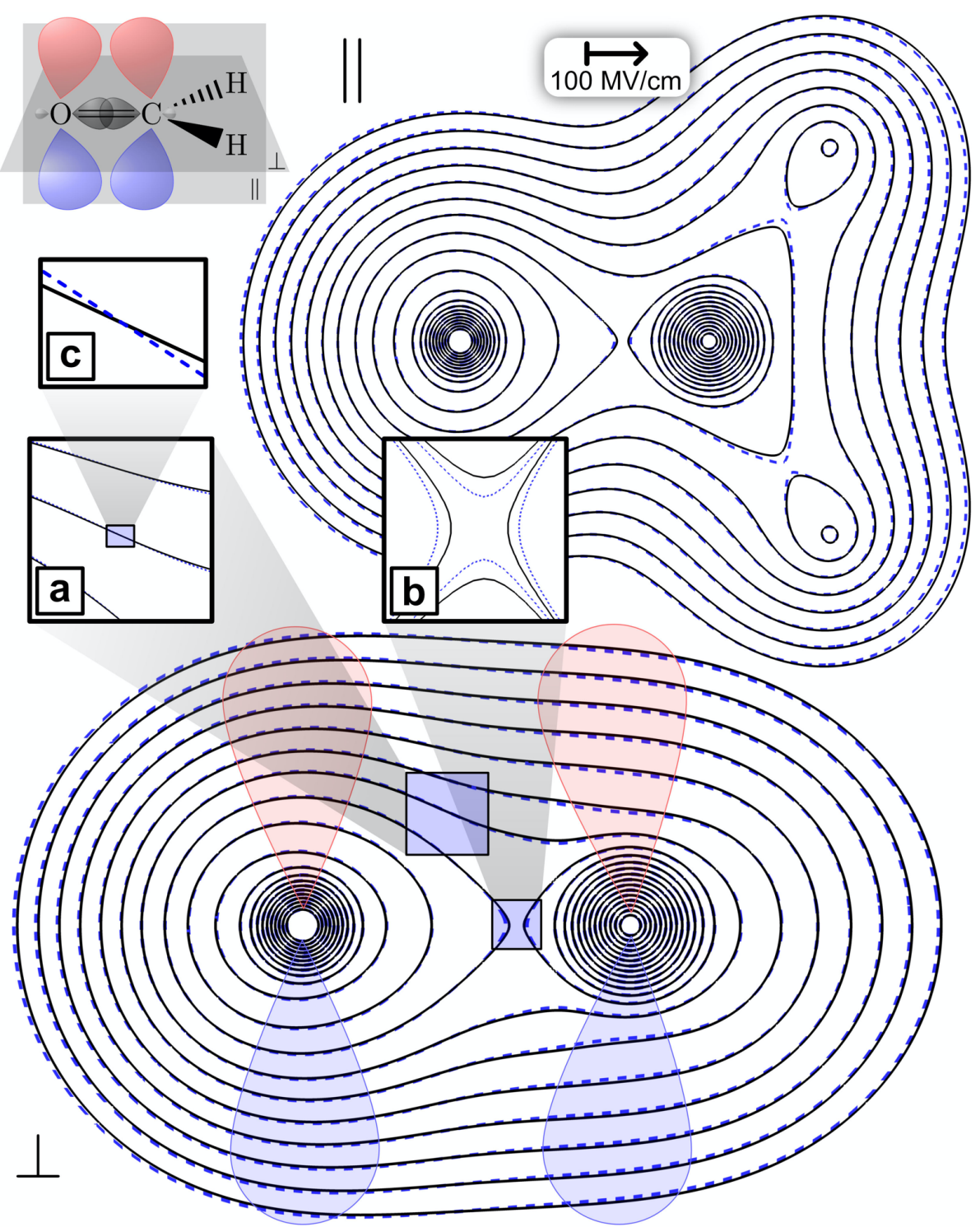

Figure 4. Contours of $\rho(r)$ in formaldehyde on planes containing the $\mathrm{C}=\mathrm{O}$ internuclear axis, parallel ॥ and perpendicular $\perp$ to the molecular plane, with and without an applied uniform external electric field. Solid black contours are those for the system with no applied field. Blue dashed contours are those resulting from a $100 \mathrm{MV} / \mathrm{cm}$ pointing towards the carbon along the molecular axis. Region $\mathrm{c}$ depicts a point of crossover between the contours with and without the external applied field. Red and blue shading on perpendicular plane depicts $\pi$-bonding $p$-orbital lobes on the $O$ and $C$ atoms through the plane. For contours close to the $O$ nucleus, i.e., for $\rho(r)>\rho\left(r_{b c p}\right)$, there is in increase in positive curvature in the $p$-orbital lobe regions, and a decrease in curvature in the $\sigma$-bonding $p$-orbital lobe regions along the $\mathrm{C}=\mathrm{O}$ axis.

Carbonyl chemistry is most frequently rationalized in terms of substituent or field induced shifts of electron density from the carbonyl $\mathrm{C}$ to the $\mathrm{O}$ atom. The $\mathrm{C}=\mathrm{O}$ bond is pictured as polar covalent with a partial negative charge on the $\mathrm{O}$ atom and a partial positive charge on the $\mathrm{C}$ atom, which renders the two atoms prone to electrophilic and nucleophilic attack respectively. 
Environments that enhance charge separation reduce the barrier to both reactions and are said to activate the carbonyl.

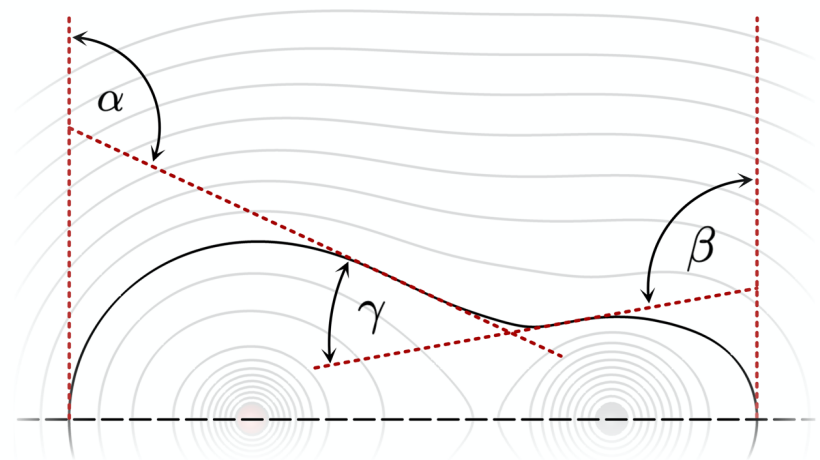

Figure 5. The dashed red lines give the tangents to the bold contour at the terminal ends of the molecule and at the ZGC points marking the boundaries between the trough and corner regions of the $\mathrm{CO}$ molecule. Thus, the total curvature on specified contour in the corner region on the $O$ atom is given by twice the angle $\alpha$, the total positive curvature on the $C$ atom is given by twice the angle $\beta$, and the total negative curvature on this contour is given by twice the angle $\gamma$.

KSI is one of many enzymes in which a carbonyl plays an active part. It has been shown that an external electric field pointing from the $\mathrm{O}$ to the $\mathrm{C}$ atom along the relevant $\mathrm{C}=\mathrm{O}$ bond path lowers an activation barrier to the catalytic reaction [15-19]. Consistent with the general mechanism of carbonyl activation, the field is presumed to enhance charge separation by pushing electron density from the $\mathrm{C}$ to the $\mathrm{O}$ atom.

This observation provides an ideal starting point in our effort to apply geometric principles to a chemical process, specifically the activation of a carbonyl by an applied external electric field. A formaldehyde molecule serves as an initial model for this process.

Figure 4 depicts the response of the electron density contours of formaldehyde in the molecular plane and in the perpendicular mirror plane when subjected to a $100 \mathrm{MV} / \mathrm{cm}$ electric field applied parallel to the $\mathrm{C}=\mathrm{O}$ internuclear axis. Note that the field magnitude is motivated by the fields estimated to be produced by the protein macromolecules and act on the bound carbonyl within the KSI binding site [16]. From a chemical perspective these planes are particularly relevant as the $\mathrm{C}=\mathrm{O}$ bond achieves its maximum $\sigma$-overlap in the molecular plane and its maximum $\pi$-overlap in the perpendicular mirror plane.

Not surprisingly, the contours shift as one would expect in low electron density regions where $\rho(r)$ is better approximated as a uniform electron gas. For example, on either end of the molecule the contours are displaced in the direction opposite to the applied field, with isosurfaces moving inwards toward the $\mathrm{C}$ nucleus and outwards from the $\mathrm{O}$ nucleus. However, 
counter to our initial expectations, we see little evidence of field induced charge transfer between atoms, with the $\mathrm{C}=\mathrm{O}$ bond $\mathrm{CP}$ and interatomic surface unperturbed by the field. Additionally, the change in the electron counts on the $\mathrm{O}$ and $\mathrm{C}$ atoms, 0.07 and 0.03 electrons respectively, is slight compared to charge redistribution within the $\mathrm{O}$ and $\mathrm{C}$ atoms (see discussion below and Table 1).

The charge rearrangement within atoms is apparent in the field's effects on the electron density contours. Particularly evident are the changes in the characteristic angles and the associated contours about this $\mathrm{C}=\mathrm{O}$ bond $\mathrm{CP}$ (region $\mathrm{b}$ ), where the applied field reduces both $\theta$ and $\phi$. Accordingly, the contours intersecting the bond path become less positively curved, while those intersecting the interatomic surface become more negatively curved. This increased negative curvature of the trough contours due to the applied field necessitates a corresponding increase to the curvature in the corner regions.

We can assess the magnitude of these offsetting effects by following a segment of a $\mathrm{C}=\mathrm{O}$ bond $\mathrm{CP}$ exterior contour lying in the mirror plane perpendicular to the molecular plane. The segment of interest lies between the convex CP on the C end of the molecule, its "origin," and the convex CP on the molecule's O end, its "terminus." Initially, the field contours sit inside the no-field contours but at its terminus the field contours are situated outside the no-field contours. At some point along their paths the field and no-field contours cross. We find that this crossing point occurs either on or very close to the ZGC boundary separating the $\mathrm{O}$ corner region form the trough region (e.g., in region c). Whether the ZGC boundary acts as a kind of "hinge" around which contour curvature "swings" from an applied field is a subject we are investigating. Regardless, crossing at this point makes assessing the change to total curvature in the convex region of the $\mathrm{O}$ atom undemanding.

As shown in Figure 5, the total curvature of a contour in this region is given by twice the angle $\alpha$ made by the intersection of the tangent to the contour at the ZGC point with a vector in the contour plane and perpendicular to the internuclear axis. Clearly, the crossing of the field contour from inside to outside (Figure 4, region c) increases this angle and hence the total curvature of the convex $\mathrm{O}$ region.

Our calculations reveal that all of the external field-no-field contour pairs cross from inside to outside at or near the ZGC boundary separating the convex $\mathrm{O}$ and trough regions. Therefore, the field increases the total curvature of the external contours in the corner regions of the $\mathrm{O}$ atom, which is offset by increased negative curvature in the trough region.

Turn now to the contours in the same plane but internal to the $\mathrm{C}=\mathrm{O}$ bond $\mathrm{CP}$. As 
mentioned, the field reduces the curvature of these contours where they intersect the bond path, hence there must be compensating changes at other points along the contour. Inspection of Figure 4 reveals that around the $\mathrm{O}$ atom, the compensating curvature is localized to the region occupied by $p$-orbitals participating in $\pi$-bonding.

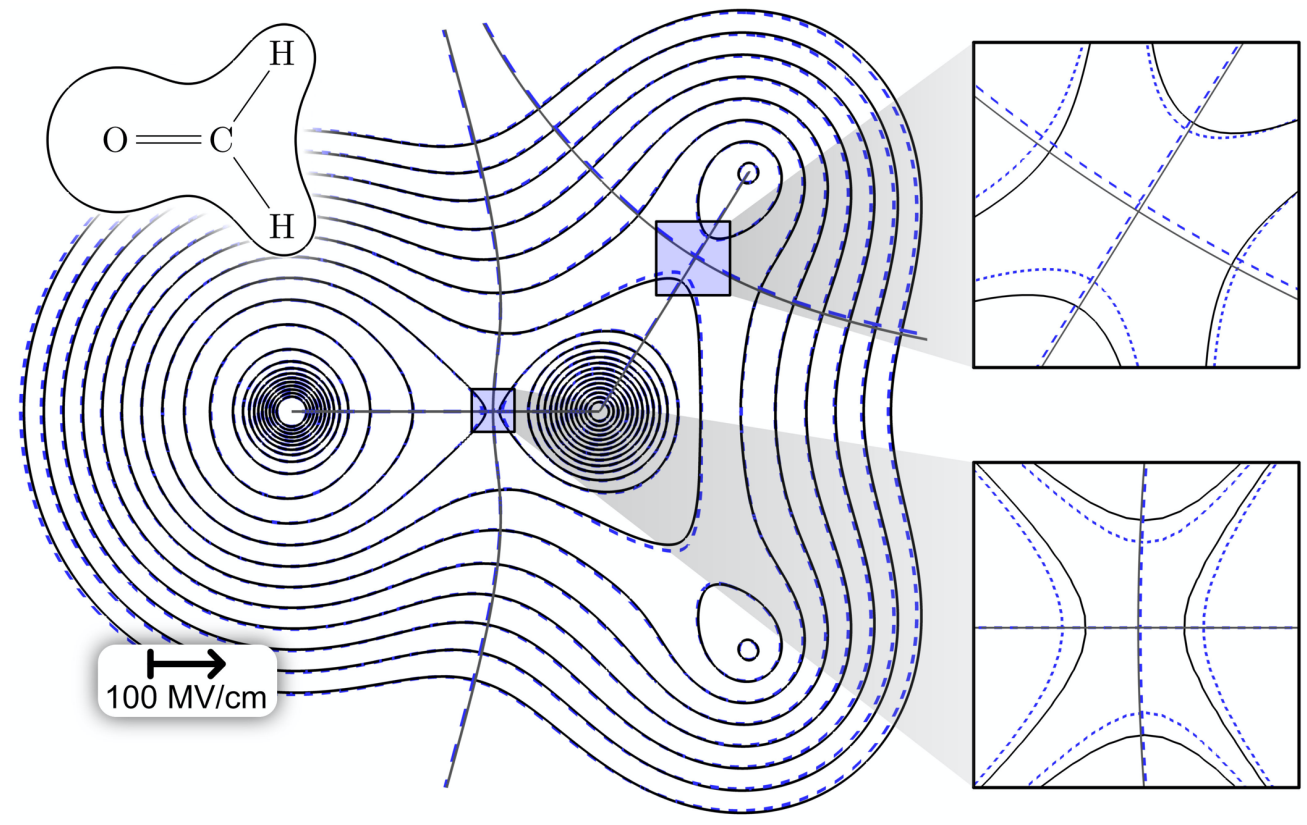

Figure 6. Contours of $\rho(r)$ in the molecular plane of formaldehyde with and without a $100 \mathrm{MV} / \mathrm{cm}$ uniform electric field applied along the $\mathrm{C}=\mathrm{O}$ internuclear axis, pointing from $\mathrm{O}$ to $\mathrm{C}$. The $\mathrm{C}-\mathrm{H}$ and $\mathrm{C}=\mathrm{O}$ bond saddle points (top and bottom right resp.) are shown in more detail with bold lines designating the interatomic surface and the lighter line the internuclear axis (bond path). The unperturbed charge density with black contours and the field induced density with dashed blue contours.

The contours around the $\mathrm{C}$ atom behave quite differently. Beginning with the response in the mirror plane perpendicular to the molecular plane, the field reduces convexity and increases negative curvature throughout the region occupied by the $\mathrm{C} p \pi$-orbitals. There appears to be a small compensating increase from field induced positive curvature along the corner line extending from the $\mathrm{C}$ nucleus to infinity. However, the bulk of the offsetting positive curvature comes from the field induced convexity on the $\mathrm{O}$ atom.

The field induced changes to the contours in the molecular plane are complicated by the fact that there are a greater number of troughs and corners over which to distribute compensating effects. Even so, the field increases at least one of the characteristic angles of the $\mathrm{C}-\mathrm{H}$ bond $\mathrm{CP}$ and thus in this plane increases the contour curvature along the $\mathrm{C}-\mathrm{H}$ bond path and flattens the contours of the corresponding trough, while having a smaller but opposite effect on the $\mathrm{C}=\mathrm{O}$ trough.

Beyond these attendant local changes to the electron density, we note similarity between 
the long-range inductive effect and the field induced responses to the contour curvatures around the $\mathrm{C}-\mathrm{H}$ and $\mathrm{C}=\mathrm{O}$ bond $\mathrm{CPs}$ (Figure 6).

Of course, the inductive effect deals with changes to the electron density in one part of a molecule due to electron donating or withdrawing groups in another part. Induction is pictured as propagating through $\sigma$ bonds linking one region of a molecule to another.

In this context, consider how the effects of an applied field propagate through neighboring bonds of formaldehyde. The increased characteristic angle(s) of the $\mathrm{C}-\mathrm{H}$ bond $\mathrm{CP}$ requires that the curvature of the contours interior to the double cone defining this bond also increase. These contours, though interior to the $\mathrm{C}-\mathrm{H}$ cone are exterior to the $\mathrm{C}=\mathrm{O}$ cone. This fact geometrically links the effects of the field at the $\mathrm{C}-\mathrm{H}$ and $\mathrm{C}=\mathrm{O}$ bond $\mathrm{CPs}$. Specifically, the field causes the curvature of the contours intersecting the $\mathrm{C}=\mathrm{O}$ bond path to decrease; which makes its external contours along the trough line more negatively curved; the increased negative curvature to these contours must now be offset with more positive curvature elsewhere along the contour; which is realized by increasing the contour curvature along the corner delineating the $\mathrm{C}-\mathrm{H}$ bond path. We designate this type of behavior as out-of-phase coupling - with the obvious implication that in-phase coupling occurs when the characteristic angles on neighboring bond CPs change in the same direction.

Whether a perturbation induces in-phase or out-of-phase coupling appears to be solely determined by the relative values of the electron density at neighboring critical points. When these values are equivalent, as for example along the $\mathrm{C}$ backbone of an alkane, neighboring critical points will couple in-phase, otherwise, neighboring bonds will couple out-of-phase. We hypothesize (supported by preliminary calculations) that the magnitude of the out-of-phase response will scale with the difference in the value of the charge density at the neighboring bond CPs. Regardless, as this coupling effect propagates from neighbor to neighbor, it is possible to alter the field induced response around a particular bond by altering $\rho\left(r_{c p}\right)$ of a distant bond, which could be achieved with substituents-adding electron donating or withdrawing groups_ — or simply by altering bond lengths via steric effects.

Returning to the broader question regarding the overall changes to the isosurfaces from the applied field: In the planes examined, the cumulative effect of the field is to increase total Gaussian curvature on the $\mathrm{O}$-atom at the expense of the $\mathrm{C}$ atom. The resulting curvaturepolarization produces a deeper trough separating the $\mathrm{O}$ and $\mathrm{C}$ atoms through increased convexity in the region coincident with $\mathrm{O} p \pi$-orbitals that is offset by a contraction of the corresponding region on the $\mathrm{C}$ atom. The hyperbolic points on the isosurfaces defining the 


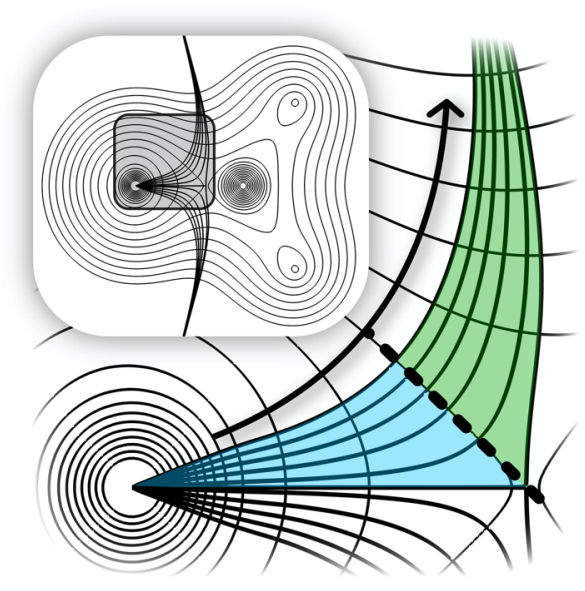

Figure 7. Contours of $\rho(r)$ in the molecular plane of formaldehyde with a set of gradient paths originating at the $\mathrm{O}$ nuclear $\mathrm{CP}$ at bottom-left. Moving down a pair of neighboring paths in the corner (cyan) region sweeps out an increasingly wider area, while in a trough region (green), neighboring paths sweep out an increasingly narrow area.

These observations, drawn from an inspection of the contours in just two planes, are suggestive of more spherical $\mathrm{C}$ and $\mathrm{O}$ atoms resulting from a field induced reduction to the $\mathrm{C}=\mathrm{O}$ bond $\pi$ character. Fortunately, we may move beyond simply "suggestive" to a more definitive statement of the field's effects by using gradient bundle analysis (GBA) to calculate the global curvature distribution and its field induced change [20-24].

GBA is a natural extension of QTAIM. As Bader commented [7], "further study of the gradient vector field of the electron density leads to a complete theory of structure and structural stability." In accordance with this comment, GBA provides the tools to holistically analyze $\nabla \rho(r)$.

Just as QTAIM defines a nonarbitrary partitioning of a molecule into Bader atoms bounded by ZFSs, GBA further partitions Bader atoms into gradient bundles (GBs) bounded by ZFSs. Where Bader atoms impose a global geometry on a molecule and allow one to unambiguously determine the energy related properties of its atoms, GBs impose a local geometry which allows one to determine the continuous distribution of these properties within an atom.

By way of illustration Figure 7, depicts a gradient field that originates at the O-atom and sweeps out an area that covers the $\mathrm{O}$ side of the $\mathrm{C}=\mathrm{O}$ trough of formaldehyde. In this $2 \mathrm{D}$ simplification, at the $\mathrm{O}$ nucleus, where all gradient paths are radial, an individual gradient path can be specified by a single angle, say $\theta$. The field is constructed so that the angular distance between neighboring gradient paths is held constant at some value $d \theta$. 
The area bounded by neighboring paths is a GB. Its boundary is a ZFS, hence its energy and energy-related properties are well-defined. However, these properties are defined only as the integral over the entire GB. As the angle $d \theta$ approaches zero, a GB's properties become functions of the single variable $\theta$.

It has been demonstrated that the area of a gradient bundle is determined only by the curvature of the contours it intersects [25]. Conceptually this makes sense for as we proceed along neighboring gradient paths, they diverge in regions where the contour curvatures are positive (corners) and converge where the contour curvatures are negative (troughs). Hence, gradient bundle area serves as a measure of total contour curvature along gradient paths.

The extension to $3 \mathrm{D}$ is straightforward. We construct a gradient field consisting of a conceptually infinite number of gradient paths (in practice more than 20,000) uniformly distributed around each nuclear CP. Each of these paths may be specified by a pair of angular spherical coordinates $(\theta, \phi)$ that give the direction of the gradient near the nucleus, where all gradient paths are radial. At some distance from the nucleus these paths are no longer radial and in fact may be space curves characterized by both curvature and torsion. A differential gradient bundle is now defined as an infinitesimal differential volume element with, in the simplest case, a triangular cross section and edges defined by three gradient paths. By integrating some 3D geometric or field variable within a differential gradient bundle, say $\rho(r)$, one gets a $2 \mathrm{D}$ "condensed" function $\mathcal{P}(\theta, \phi)$ that depends only on $\theta$ and $\phi$, the variation along the gradient having been integrated out. With condensed properties depending only on $\theta$ and $\phi$, it is convenient to represent their values as contours mapped onto a sphere.

Condensed properties have units of the input property per steradian $(s r)$ or square radian. Thus $\mathcal{P}(\theta, \phi)$ has atomic units of electrons/sr; condensed energy, $\mathcal{E}(\theta, \phi)$, of $E_{h} / s r$; and condensed volume, $\mathcal{V}(\theta, \phi)$, units of $a_{0}^{3} / s r$. Integrating a condensed property over all $\theta$ and $\phi$ recovers the corresponding Bader atom property, e.g., integrating $\mathcal{P}(\theta, \phi), \mathcal{E}(\theta, \phi)$, and $\mathcal{V}(\theta, \phi)$ recovers the Bader atom's electronic population, energy and volume respectively.

The condensed volume is descriptive of the charge density's intrinsic geometry, as can be seen from the behavior of the gradient paths depicted in Figure 7. The paths bounding a GB diverge as they pass through regions of convexity, hence increasing GB volume. When passing through concave regions the opposite is true, with neighboring gradient paths converging and reducing gradient bundle volume. When passing through saddle regions GBs expand in one direction and contract in the perpendicular direction. GB volume is determined only by the Gaussian curvature of the contours contained in the gradient bundle [25]. Hence, 
$\mathcal{V}(\theta, \phi)$ serves as a measure of total isosurface curvature along gradient paths and is solely determined by local geometry.

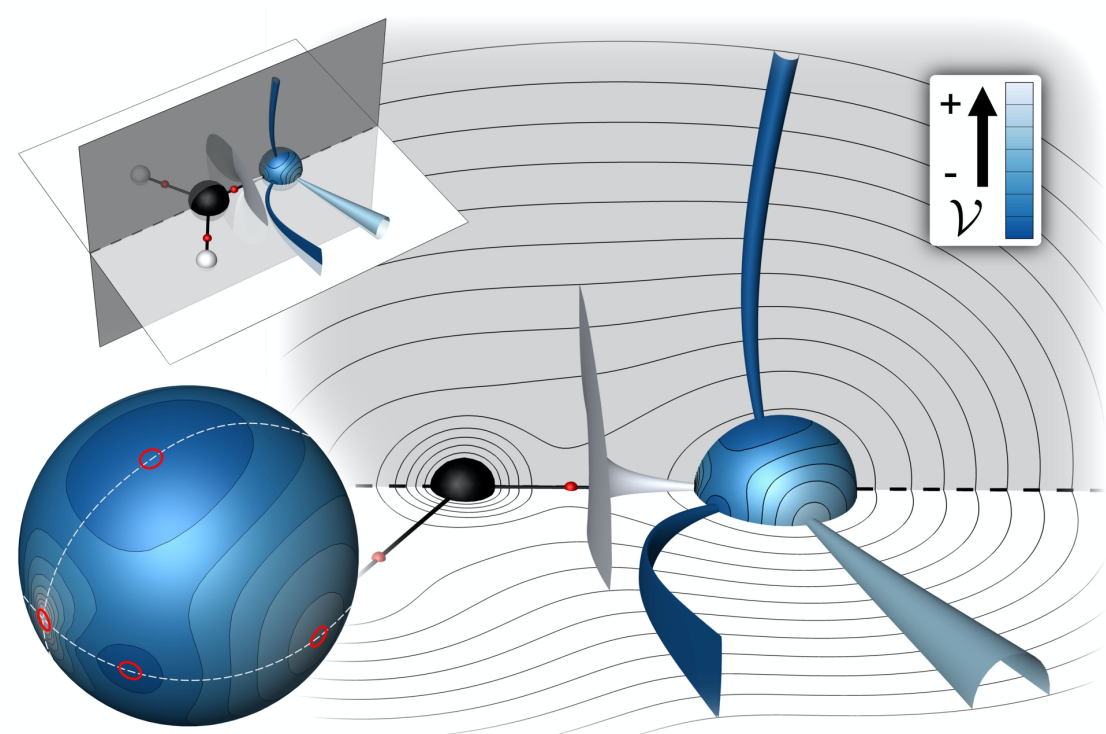

Figure 8. The condensed volume of the $\mathrm{O}$ atom of formaldehyde (left) shown with representative gradient bundles (right).

As a means of clarifying this issue, the condensed volume for the $\mathrm{O}$ atom of formaldehyde is represented in Figure 8, with the integrated condensed volume mapped onto a sphere and depicted in the lower left of the figure.

Just as QTAIM analysis gives special consideration to the CPs of $\rho(r)$ as representative of electron density topology, GBA takes particular notice of extremal points of $\mathcal{V}(\theta, \phi)$. In this way the complexity of $\nabla \rho(r)$ and its geometry can be rationalized in terms of the behavior of a few special GBs about every atom of a molecule. Accordingly, also shown in Figure 8 and designated with red circles are four $\mathcal{V}(\theta, \phi)$ extremal points, two maxima and two minima.

The gradient bundles corresponding to these extremal regions are shown on the right of Figure 8. Note that the maxima gradient bundles lie for the most part along corners while minima intersect isosurfaces near point of ZGC. Still, the gradient bundle containing the $\mathrm{C}=\mathrm{O}$ bond path diverges umbrella-like near the interatomic surface and takes in volume from the $\mathrm{C}=\mathrm{O}$ trough region. The other maximum lies along what is essentially the $\mathrm{O}$ lone pair region. The in-plane $\mathcal{V}(\theta, \phi)$ minimum is noteworthy as it appears to nearly coincide with the ZGC boundary separating the $\mathrm{C}=\mathrm{O}$ trough from the $\mathrm{O}$ corner regions (see Figure $4 \mathrm{a}$ ).

As already demonstrated, an activating electric field alters isosurface curvature and hence 

(blue).

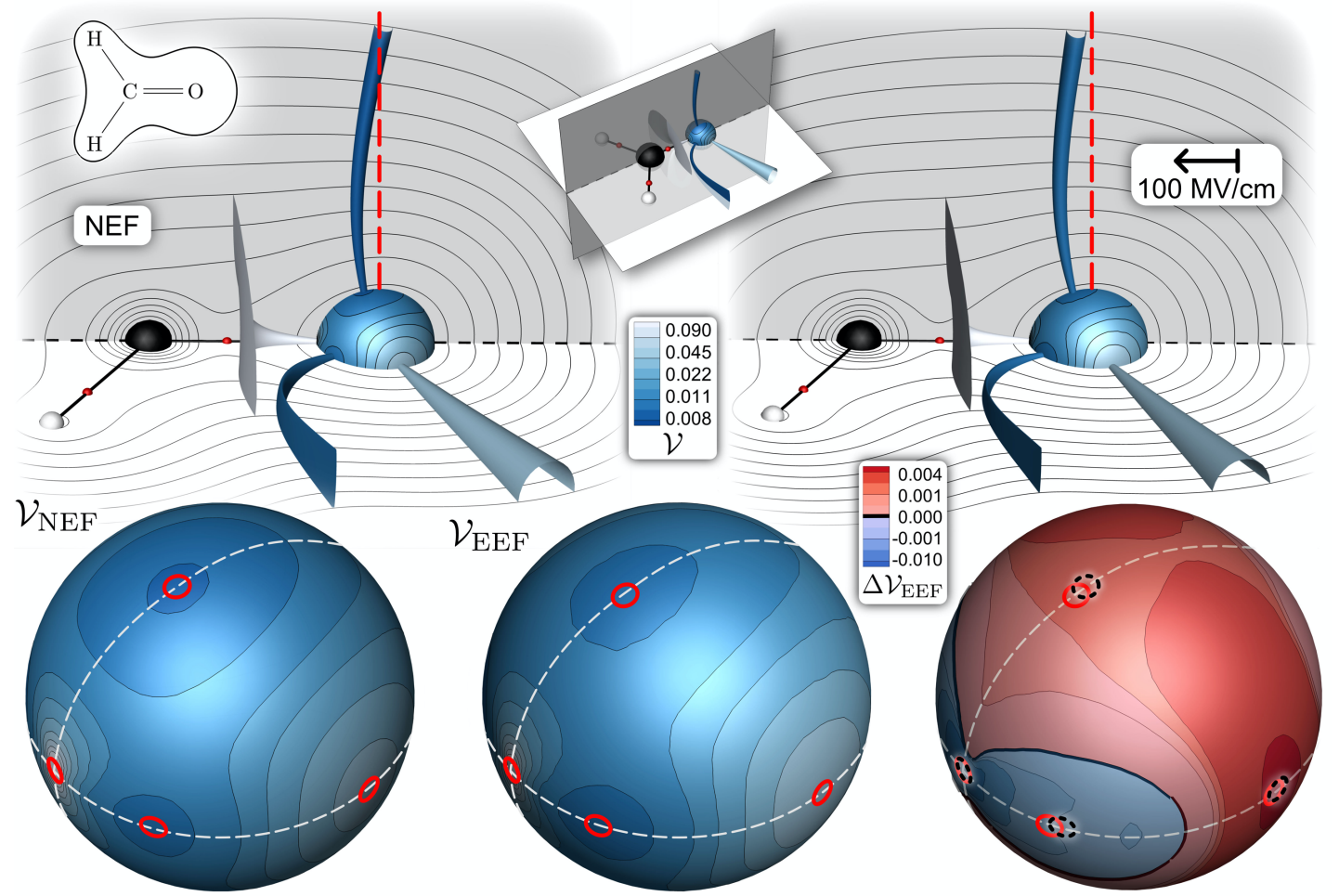

Figure 9. The GB condensed volume of formaldehyde in the no field situation (left), due to an applied field (center), and their difference (right). Red shading indicates GBs where the field has increased volume by inducing more corner character, while blue shading indicates a loss of GB volume.

The field's effects are subtle but noticeable, with small shifts in the locations of the $\mathcal{V}(\theta, \phi)$ CPs. The condensed volume difference map brings these differences into stark relief. Most dramatic is the increase in corner character and the corresponding expansion of GB volume in the $\mathrm{O}$ lone pair regions. The increased curvature in this region is achieved through an increase in negative Gaussian curvature in the trough regions. (Recall that GBs passing through regions of more negative curvature contract more rapidly and hence lose volume.)

A more detailed and quantitative analysis of field induced curvature redistribution is afforded by considering the distribution of $\mathcal{V}(\theta, \phi)$ between formaldehyde's bonding and non-bonding regions (bond bundles and lone pair bundles) identifiable using GBA [25]. Table 1 lists the total regional volumes and electron counts of formaldehyde partitioned into 
Bader atoms, and again with Bader atoms partitioned and organized into bond and lone pair regions. The bonding regions are further distinguished by the contributions to the bond from each of the bound atoms, i.e., bond wedges.

Table 1. Changes to regional volumes, $V$, and electron counts, $\rho$, in formaldehyde due to an applied external electric field of $100 \mathrm{MV} / \mathrm{cm}$ pointing from the $\mathrm{O}$ nuclear position to the $\mathrm{C}$. Atomic basins (top) and bond and lone pair regions (bottom) are truncated at the $\rho=0.001$ isosurface. Complete gradient bundle integration tables are available in the SI.

\begin{tabular}{|c|c|c|c|c|c|c|c|c|}
\hline \multirow{2}{*}{ Region } & \multicolumn{4}{|c|}{$\mathrm{V}$ [au] } & \multicolumn{4}{|c|}{$\rho[\mathrm{e}]$} \\
\hline & NEF & EEF & $\Delta_{\mathrm{EEF}}$ & $\% \Delta_{\mathrm{EEF}}$ & NEF & EEF & $\Delta_{\mathrm{EEF}}$ & $\% \Delta_{\mathrm{EEF}}$ \\
\hline O atomic basin & 133.0 & 139.3 & 6.2 & 4.7 & 8.92 & 8.99 & 0.07 & 0.8 \\
\hline C atomic basin & 68.2 & 68.6 & 0.5 & 0.7 & 5.06 & 5.09 & 0.03 & 0.5 \\
\hline $\mathrm{H}(\mathrm{x} 2)$ & 50.3 & 46.3 & -4.0 & -7.9 & 0.94 & 0.90 & -0.05 & -5.0 \\
\hline Total & 301.8 & 300.5 & -1.2 & -0.4 & 15.86 & 15.87 & 0.003 & 0.021 \\
\hline $\mathrm{C}-\mathrm{H}$ bond bundle $(\mathrm{x} 2)$ & 78.2 & 75.4 & -2.9 & -3.7 & 2.82 & 2.84 & 0.02 & 0.8 \\
\hline$\llcorner C$ bond wedge & 28.0 & 29.1 & 1.1 & 4.0 & 1.88 & 1.95 & 0.07 & 3.7 \\
\hline$\llcorner\mathrm{H}$ bond wedge & 50.3 & 46.3 & -4.0 & -7.9 & 0.94 & 0.90 & -0.05 & -5.0 \\
\hline $\mathrm{C}=\mathrm{O}$ bond bundle & 34.7 & 32.6 & -2.1 & -5.9 & 3.55 & 3.40 & -0.14 & -4.1 \\
\hline$\llcorner C$ bond wedge & 12.2 & 10.5 & -1.7 & -14.3 & 1.31 & 1.20 & -0.11 & -8.7 \\
\hline$\llcorner\mathrm{O}$ bond wedge & 22.4 & 22.1 & -0.3 & -1.4 & 2.24 & 2.21 & -0.03 & -1.3 \\
\hline O lone pair bundle $(x 2)$ & 55.3 & 58.6 & 3.3 & 5.9 & 3.34 & 3.39 & 0.05 & 1.5 \\
\hline Total & 301.8 & 300.5 & -1.2 & -0.4 & 15.86 & 15.87 & 0.004 & 0.022 \\
\hline
\end{tabular}

Beginning with the atomic basins. The field induces curvature polarization that increases the volume (curvature) of the $\mathrm{O}$ atom by $4.7 \%$, but not by depleting the $\mathrm{C}$ atom but rather the $\mathrm{H}$ atoms. This curvature polarization is weakly reflected in the electron counts by a small charge transfer from the $\mathrm{H}$-atoms to the $\mathrm{O}$ and $\mathrm{C}$ atoms. These effects are consistent with the observed field induced expansion and contraction of low-density contours at the ends of the molecule in Figure 4.

Moving on to a rigorous analysis of the curvature polarization within atoms. There is an increase to the volume of the $\mathrm{O}$ lone pairs that is offset by depletion in the $\mathrm{C}-\mathrm{H}$ and $\mathrm{C}=\mathrm{O}$ bonds. A substantive part of this depletion comes from the $\mathrm{C}$ atom's contribution to the $\mathrm{C}=\mathrm{O}$ bond (volume decrease of 14.3\%) and a corresponding loss of 0.11 electrons. At the same time, there is an increase to the volume of the $\mathrm{C}-\mathrm{H}$ bond from the $\mathrm{C}$ bond wedge.

Putting these observations into more familiar chemical terms. These changes are consistent with a loss of $\mathrm{C}$ atom $\pi$-bonding character, which is offset by an increase in $\sigma$ character directed principally toward the $\mathrm{H}$ atoms.

Altogether, the tabulated values, especially regional volumes, paint a concise and chemically relevant picture of formaldehyde's response to an electric field. From these results we conclude that a primary indicator of carbonyl activation is field induced curvature 

pair regions.

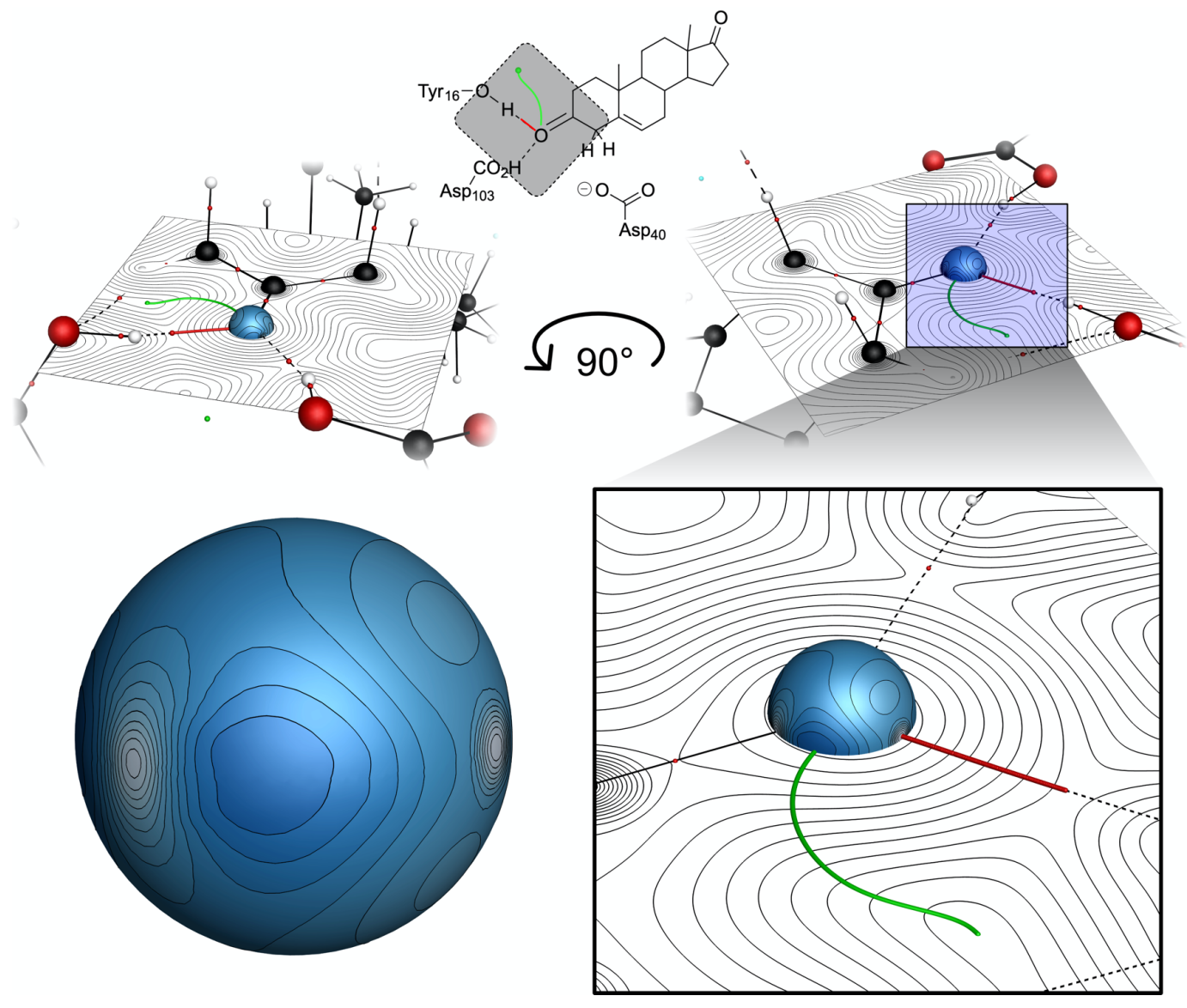

Figure 10. The condensed volume about the carbonyl oxygen atom in KSI, mapped onto a sphere centered at the oxygen nuclear critical point. Contours on the sphere indicate low (blue) to high (white) values of condensed mean curvature (bottom-left). The active site of KSI is depicted at top with the plane and gradient paths of interested indicated. The middle row consists of two different angles of the active site with contours of $\rho(r)$ on the indicated plane along with two gradient paths: one leaving the sphere at a minimum and terminating at a ring critical point (green), and the other at a maximum and terminating at an $\mathrm{O}-\mathrm{H}$ bond critical point (red). At bottom-right is a zoomed image of the sphere and the gradient paths.

We now turn to a GBA of the carbonyl of KSI - an enzyme where the intramolecular electric field is known to activate the bound carbonyl. While a more complete gradient bundle analysis of KSI is available (cite to be added), here we focus exclusively on the carbonyl $\mathrm{O}$ atom. Enzyme efficiency in general and KSI specifically results from the perfect positioning of residues in and around the active site [26] (Figure 10). For KSI, notable are the Tyr 16 and $\mathrm{Asp}_{103}$ residues forming hydrogen bonds with the substrate carbonyl $\mathrm{O}$ atom. Conventional wisdom attributes carbonyl activation through hydrogen bonding to a density shift from the $\mathrm{O}$ 
to the $\mathrm{H}$ atom, which we do not observe. However, there are changes to isosurface curvature consistent with those associated with the field induced activation of formaldehyde. Most pronounced is the deepening of the $\mathcal{V}(\theta, \phi)$ minimum indicative of greater trough character along the boundary separating the maxima of $\mathrm{C}=\mathrm{O}$ bond path and the $\mathrm{O}-\mathrm{H}$ bond with $\mathrm{Tyr}_{16}$ (the lone pair maximum of formaldehyde). This increased trough character is made possible by more corner character along the $\mathrm{O}-\mathrm{H}$ bond path. However, as a similar change in trough/corner character is not observed to result from the hydrogen bond with Asp $_{103}$, it is not the hydrogen bond per se that is responsible for these significant curvature changes. Rather, the hydrogen bond between the carbonyl $\mathrm{O}$ and $\mathrm{Tyr}_{16}$ is part of a hexagonal ring that must be accompanied by a depression line and a concave region above and below the ring. GBs passing through depressions are necessarily low volume, thus allowing for greater corner character between the carbonyl $\mathrm{O}$ and $\mathrm{Tyr}_{16}$. The net result is a deepening of the $\mathrm{C}=\mathrm{O}$ trough and yielding this interaction more spherical.

Thus, we have a consistent picture for the mechanism of carbonyl activation as resulting from interactions that increase corner character on the side of the $\mathrm{O}$ atom opposite the $\mathrm{C}=\mathrm{O}$ bond $\mathrm{CP}$, which is compensated through increased negative curvature in the $\mathrm{C}=\mathrm{O}$ trough.

\section{Summary}

One of the challenges facing chemistry is the integration of first principles calculations with molecular design and synthesis. This task is complicated by the fact that much of synthesis' mechanistic foundation derives from early $20^{\text {th }}$ century heuristic concepts predating quantum mechanics [27-29]. Perhaps the most fundamental of these are those dealing with electron mobility, which is often represented with the curvy arrow formalism introduced in 1922 by Kermack and Robinson [29]. For nearly a century, the supposed mechanisms governing electron redistribution and related phenomena have been empirically tuned to account for an ever-greater number of chemical reactions. And now, taken together, these mechanisms comprise a predictive formalism that is incredibly useful when designing synthetic strategies. However, at best they have obscure quantum mechanical connections [30]. In the absence of such connections, first principles methods provide synthetic chemists with limited intuitive advantages.

Here we have begun the process of reframing the traditional view of carbonyl activation, thought to result from enhanced charge separation, in the quantum mechanically and 
mathematically rigorous terms of charge density differential geometry. Specifically, rather than dramatic shifts of electron density, activation results from subtle variations of $\nabla \rho(r)$ that can be quantified by the changes to isosurface curvature along gradient paths. An attractive facet of this new perspective is that allowed changes to isosurface curvature are constrained by rigorous mathematical principles. If these findings can be shown to apply to a broader range of chemical reactions, then much of our hard-won mechanistic understanding of chemical reactions will receive a $21^{\text {st }}$ century update.

\section{Computational methods}

All DFT calculations were performed with the Amsterdam Modeling Suite [31-33] ab initio software using the Perdew-Burke-Ernzerhof (PBE) functional [34] and a triple-zeta with polarization (TZP) all-electron basis set. Analysis was performed within the Tecplot 360 visualization package [35] using the Gradient Bundle Decomposition software of the in house Bondalyzer package by the Molecular Theory Group at Colorado School of Mines.

\section{Acknowledgement}

This work was supported by the National Science Foundation grant CHE-1903808, and by the Office of Naval Research grant N00014-10-1-0838. 


\section{References}

[1] R. F. W. Bader, Atoms in Molecules: A Quantum Theory (Clarendon Press, 1994).

[2] C. F. Matta and R. J. Boyd, editors, The Quantum Theory of Atoms in Molecules: From Solid State to DNA and Drug Design (Wiley-VCH Verlag GmbH \& Co. KGaA: Weinheim, 2007).

[3] P. L. A. Popelier, Atoms in Molecules: An Introduction (Pearson Education Limited, Essex, England, 2000).

[4] P. Nasertayoob and S. Shahbazian, Revisiting the Foundations of the Quantum Theory of Atoms in Molecules: Toward a Rigorous Definition of Topological Atoms, Int. J. Quantum Chem. 109, 726 (2009).

[5] F. Heidarzadeh and S. Shahbazian, The Quantum Divided Basins: A New Class of Quantum Subsystems, Int. J. Quantum Chem. 111, 2788 (2011).

[6] J. S. M. Anderson, P. W. Ayers, and J. I. R. Hernandez, How Ambiguous Is the Local Kinetic Energy?, J. Phys. Chem. A 114, 8884 (2010).

[7] R. F. W. Bader, P. L. A. Popelier, and T. A. Keith, Theoretical Definition of a Functional Group and the Molecular Orbital Paradigm, Angew. Chem. Int. Ed. Engl. 33, 620 (1994).

[8] J.-B. Peng, H.-Q. Geng, and X.-F. Wu, The Chemistry of CO: Carbonylation, Chem 5, 526 (2019).

[9] M. E. Eberhart, The Metallic Bond: Elastic Properties, Acta Mater. 44, 2495 (1996).

[10] M. E. Eberhart and T. E. Jones, Cauchy Pressure and the Generalized Bonding Model for Nonmagnetic Bcc Transition Metals, Phys. Rev. B 86, 134106 (2012).

[11] H. Morrison, editor, Enzyme Active Sites and Their Reaction Mechanisms (Academic Press, 2021).

[12] R. Kannan et al., Organoaluminum Cations for Carbonyl Activation, Chem. Commun. 55, 14629 (2019).

[13] Y. Román-Leshkov and M. E. Davis, Activation of Carbonyl-Containing Molecules with Solid Lewis Acids in Aqueous Media, ACS Catal. 1, 1566 (2011).

[14] P. M. Pihko, Activation of Carbonyl Compounds by Double Hydrogen Bonding: An Emerging Tool in Asymmetric Catalysis, Angew. Chem. Int. Ed. 43, 2062 (2004).

[15] W. Childs and S. G. Boxer, Solvation Response along the Reaction Coordinate in the Active Site of Ketosteroid Isomerase, J. Am. Chem. Soc. 132, 6474 (2010).

[16] S. D. Fried, S. Bagchi, and S. G. Boxer, Extreme Electric Fields Power Catalysis in the Active Site of Ketosteroid Isomerase, Science 346, 1510 (2014).

[17] Y. Wu and S. G. Boxer, A Critical Test of the Electrostatic Contribution to Catalysis with Noncanonical Amino Acids in Ketosteroid Isomerase, J. Am. Chem. Soc. 138, 11890 (2016).

[18] Y. Wu, S. D. Fried, and S. G. Boxer, A Preorganized Electric Field Leads to Minimal Geometrical Reorientation in the Catalytic Reaction of Ketosteroid Isomerase, J. Am. Chem. Soc. 142, 9993 (2020).

[19] M. R. Hennefarth and A. N. Alexandrova, Direct Look at the Electric Field in Ketosteroid 
Isomerase and Its Variants, ACS Catal. 10, 9915 (2020).

[20] M. E. Eberhart and T. E. Jones, The Two Faces of Chemistry: Can They Be Reconciled?, Found. Chem. 15, 277 (2013).

[21] A. Morgenstern et al., In Search of an Intrinsic Chemical Bond, Comput. Theor. Chem. 1053, 31 (2015).

[22] A. Morgenstern and M. Eberhart, Bond Dissociation Energies from the Topology of the Charge Density Using Gradient Bundle Analysis, Phys. Scr. 91, 023012 (2016).

[23] A. Morgenstern et al., The Influence of Zero-Flux Surface Motion on Chemical Reactivity, Phys. Chem. Chem. Phys. 18, 5638 (2016).

[24] A. Morgenstern, T. R. Wilson, and M. E. Eberhart, Predicting Chemical Reactivity from the Charge Density through Gradient Bundle Analysis: Moving beyond Fukui Functions, J. Phys. Chem. A 121, 4341 (2017).

[25] T. R. Wilson et al., Observing the 3D Chemical Bond and Its Energy Distribution in a Projected Space, ChemPhysChem 20, 3289 (2019).

[26] A. Warshel, Electrostatic Basis of Structure-Function Correlation in Proteins, Acc. Chem. Res. 14, 284 (1981).

[27] G. N. Lewis, THE ATOM AND THE MOLECULE., J. Am. Chem. Soc. 38, 762 (1916).

[28] G. N. Lewis, Valence and the Structure of Atoms and Molecules (The Chemical Catalogue Company: New York, 1923).

[29] W. O. Kermack and R. Robinson, LI.-An Explanation of the Property of Induced Polarity of Atoms and an Interpretation of the Theory of Partial Valencies on an Electronic Basis, J Chem Soc Trans 121, 427 (1922).

[30] J. Andrés et al., Curly Arrows, Electron Flow, and Reaction Mechanisms from the Perspective of the Bonding Evolution Theory, Phys. Chem. Chem. Phys. 19, 29031 (2017).

[31] G. te Velde et al., Chemistry with ADF, J. Comput. Chem. 22, 931 (2001).

[32] C. Fonseca Guerra et al., Towards an Order-N DFT Method, Theor. Chem. Acc. 99, 391 (1998).

[33] E. J. Baerends et al., (2016).

[34] J. P. Perdew, K. Burke, and M. Ernzerhof, Generalized Gradient Approximation Made Simple, Phys. Rev. Lett. 77, 3865 (1996).

[35] Tecplot Inc., Tecplot $3602013 R 1$ (Tecplot Inc., 2013). 